\title{
Determinación del espesor óptico de aerosol en la estación antártica peruana Machu Picchu
}

\section{Determination of aerosol optical thickness in the Peruvian Antarctic Base Machu Picchu}

\author{
Ana Contreras ${ }^{1}$, Julio Ángeles ${ }^{2}$, Luis Suárez ${ }^{3}$, José Flores ${ }^{4}$ \\ http://dx.doi.org/10.21503/CienciayDesarrollo.2014.v17i1.02
}

\section{RESUMEN}

Objetivos: Determinar el espesor óptico de los aerosoles (EOA) durante los meses de enero 2007 y 2008 y febrero de 2013, en la Estación Científica Antártica Machu Picchu (ECAMP). Métodos: La investigación fue descriptiva comparativa. El principal instrumento para evaluar el EOA fue el fotómetro solar SP02-L para mediciones directas del sol con 4 sensores centrados en las longitudes de onda 412, 500, 675 y $862 \mathrm{~nm}$. Solo fueron utilizados registros con un ángulo cenital solar inferior a $80^{\circ}$ y con cero de cobertura nubosa $30^{\circ}$ alrededor del sol. Estas mediciones fueron comparadas, a través de la prueba de Pearson, con EOA a $550 \mathrm{~nm}$ de los sensores satelitales MODIS (MOderate Resolution Imaging Spectroradiometer). Resultados: Se determinó una máxima y mínima media de EOA de 0,1061 y 0,0616, respectivamente. Se reportó un coeficiente de correlación con el sensor MODIS, tanto del satélite Aqua y Terra, siendo $0,0284$ y 0,0059 ( $p<0,05)$, respectivamente. La misma correlación fue evaluada con el Índice de Aerosol del sensor OMI resultando una mayor correlación, $0,3606(\mathrm{p}<0,05)$. Comparando el EOA resultante en la ECAMP con las demás estaciones antárticas se encontraron valores similares, especialmente con los cercanos a las zonas costeras como Neumayer y Aboa. Conclusiones: Fue posible implementar una estación de monitoreo que provea una primera evaluación de las propiedades de los aerosoles en la ECAMP y su relación con el monitoreo satelital. Ello también contribuirá al estudio del forzamiento radiativo y a las propiedades ópticas de los aerosoles.

Palabras clave: Espesor óptico de aerosol, aerosol, Antártida.

\section{ABSTRACT}

Objectives: To determine the aerosol optical thickness for the months of January 2007 and 2008 and February 2013, Antarctic Scientific Base Machu Picchu. Methods: The research was a descriptivecomparative method. The main instrument to evaluate the EOA was SP02-L sunphotometer for direct measurements of the Sun with 4 sensors centered at wavelengths $412,500,675$ and $862 \mathrm{~nm}$. Records were only used with a solar Zenith angle less than $80^{\circ}$ and zero cloud coverage $30^{\circ}$ around the Sun. These measurements were compared through Pearson test with aerosol optical thickness at $550 \mathrm{~nm}$ from MODIS satellite sensors (MOderateResolutionImagingSpectroradiometer). Results: The mean maximum and minimum of 0.1061 and 0.0616 of aerosol optical thickness respectively were determined. A correlation coefficient with the MODIS sensor, both Terra and Aqua satellite was reported, being 0.0284 and 0.0059 $(\mathrm{p}<0.05)$, respectively. The same correlation was assessed with the OMI sensor Aerosol Index resulting

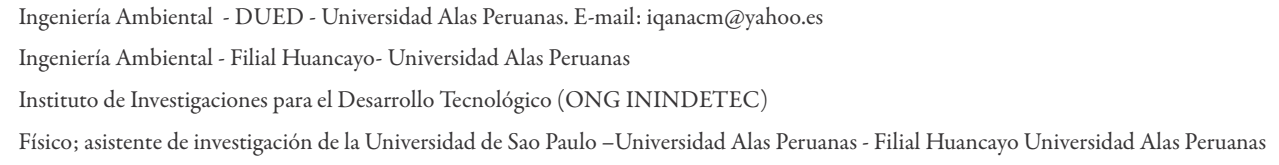


in a higher correlation, $0.3606(\mathrm{p}<0.05)$. Comparing the resulting aerosol optical thickness at Antarctic Scientific Base Machu Picchu with other Antarctic basis were found similar values especially with the near coastal areas as Neumayer and Aboa. Conclusions: It was possible to implement a monitoring station to provide a first assessment of the properties of aerosols in the Antarctic Scientific Base Machu Picchu and its relationship with the monitoring satellite. This study also contributes to radiative forcing and optical properties of aerosols.

Keywords: Aerosol optical thickness, aerosol, Antarctic.

\section{INTRODUCCIÓN}

Los aerosoles atmosféricos o material particulado, son un conjunto de partículas gaseosas, sólidas o líquidas suspendidas en la atmósfera (1) y juegan un rol importante en el cambio climático (2), porque influyen en el clima de manera directa mediante la dispersión y absorción de la radiación solar produciendo el enfriamiento y calentamiento, respectivamente (3); y de manera indirecta, el tamaño de los aerosoles actúan en la formación de distintos tipos de nubes, ya que facilitan el proceso de condensación de vapor de agua en la atmósfera actuando como núcleos de condensación para la formación de nubes o modificando las propiedades ópticas y período de vida de las nubes (4).

Es tanto su importancia que la Organización Meteorológica Mundial (OMM) ha incluido el espesor óptico de aerosoles en el listado básico de medidas del Programa de Vigilancia Atmosférica Mundial ya que alteran en forma directa el clima debido a la dispersión y absorción de la radiación y la influencia en el albedo planetario en el sistema climático. Los efectos indirectos incluyen la influencia de los aerosoles antropogénicos en núcleos de condensación para formación de nubes (5).

Los aerosoles también pueden producir un gran efecto en la salud, existe abundante evidencia que muestra una fuerte correlación estadística positiva entre la concentración de PM10 y la mortalidad diaria (6), y entre PM10 y las enfermedades respiratorias agudas bajas en niños menores de 14 años (7).

Aún no se conoce la cantidad de aerosoles contribuidos por los humanos en la región antártica peruana; sin embargo, existen estudios que demuestran que el transporte de contaminantes del aire pueden alcanzar sitios tan alejados como la Antártida (2), y que la composición química varía mucho de unas partículas a otras, dependiendo del origen y pudiendo ser trasladados a grandes distancias por acción de los vientos. Por esta razón, es de interés el estudio de los aerosoles para identificar una influencia del transporte de contaminantes e identificar las fuentes más importantes o algún efecto antropogénico negativo en la calidad de aire (8) y comparar con las mediciones de otras estaciones antárticas que están en la zona costera para lograr uniformizar la metodología en la determinación del EOA (9).

El problema que conduce al presente trabajo es: ¿Cuál es la variabilidad del espesor óptico de aerosol existente en los meses de enero del 2007 y 2008 y febrero del 2013 en la Estación Antártica Peruana Machu Picchu?

El objetivo es evaluar la variabilidad del espesor óptico de los aerosoles precisamente en tales meses, a raíz de la XVII, XVIII y XXI Expedición Antártica Peruana realizada durante las referidas fechas en la Estación Científica Antártica Machu Picchu (ECAMP).

El trabajo busca con ello es determinar las prin- 
cipales propiedades ópticas de los aerosoles y desarrollar una evaluación para comprobar si los aerosoles contribuyen a un enfriamiento o calentamiento en el sistema terrestre, sea para mejorar el entendimiento de los impactos de su variación como para implementar sus valores en modelos climatológicos, puesto que el estudio de los aerosoles aún tiene un bajo nivel de entendimiento (2)

\section{MATERIAL Y MÉTODOS}

Las mediciones se dieron en la estación científica Antártica Peruana Machu Picchu (ECAMP) ubicada en la Punta Crespín, Ensenada Mackellar, Isla Rey Jorge (6205’30”'S y 58²8'16” W a 6 m.s.n.m) durante los meses de enero y febrero para los años 2007(ANTAR XVII), 2008(ANTAR XVIII) y 2013 (ANTAR XXI).

Para la determinación del espesor óptico de aerosol (EOA), se utilizó un fotómetro solar SP02-L (Middleton Inc. Australia) (10); este instrumento es utilizado para observaciones de aerosoles, ozono y vapor de agua. Para la configuración del aerosol, el SP02-L tiene 4 canales en las longitudes de onda de 412, 500, 675 y $862 \mathrm{~nm}$, correspondientes a la radiación visible. Estos espectro-piroheliometros se encuentran axialmente alineados en un recinto herméticamente cerrado, los mismos que actúan de modo simultáneo cuando el instrumento es alineado directamente al sol, acción que permite una resolución fina de indicación del espesor óptico de aerosol en la atmósfera.

La versión SP02-L tiene un ancho de banda de $10 \mathrm{~nm}$ y un campo de visión de $2.5^{\circ}$, las señales de salida tienen un rango de -0.05 a 4.50 VDC, adicionalmente tiene una salida de temperatura interna $\left(10 \mathrm{mV} / 1^{\circ} \mathrm{C}\right)$. La temperatura de operación del fotómetro solar es de $-30^{\circ} \mathrm{C}$ a $70{ }^{\circ} \mathrm{C}$.

También se recurrió a los satélites Aqua y Terra que cuentan con el sensor MODIS (Modera- te Resolution Imaging Spectroradiometer) que nos brindan el EOA a $550 \mathrm{~nm}$ perteneciente al área de $61,68^{\circ}$ a $62,68^{\circ}$ latitud Sur y al de $58,4^{0}$ a 59, $4^{0}$ longitud Oeste.

Las concentraciones de la columna total de ozono para la determinación del espesor óptico de aerosol se obtuvieron del sensor OMI (Ozone Monitoring Instrument) de la NASA que brinda la variable denominada índice de aerosol (IA) desde el año 2004.

\section{Espesor óptico de aerosol (EOA)}

El EOA como se indica en la ecuación 1 se estima a partir del espesor óptico total (), el cual es la suma de los espesores ópticos del aire (referido como dispersión Rayleigh, $\left(\mathbf{t}_{\text {Rayleigh }}\right)$, ozono $\left(\mathbf{t}_{\text {ozono }}\right)$ y aerosoles (EOA) (11). Este cálculo se determina para cada una de las longitudes de onda correspondientes al fotómetro solar SP02L, siendo 412, 500, 675 y $862 \mathrm{~nm}$.

$$
E O A=\tau-\tau_{\text {Rayleigh }}-\tau_{\text {ozono }}
$$

La secuencia de ecuaciones se basa en el cálculo de los tres primeros espesores ópticos. La determinación del espesor óptico total está basada en la Ley de Lambert-Beer (ecuación 2), que explica la atenuación de la radiación solar al paso por la atmósfera (9).

$$
\tau=\frac{\ln \left(R I_{0 \lambda} / I_{\lambda}\right)}{m}(2)
$$

Donde $\mathrm{R}$ es el factor de corrección distancia soltierra; es la intensidad de radiación observada a una longitud de onda $(\lambda)$, a nivel de la superficie del suelo; es la intensidad de radiación en el tope de la atmósfera, obtenido gráficamente por el método de Langley (12), para una $\lambda$ en parti- 
cular; $y m$ es la masa del aire determinado por la secante del ángulo cenit solar.

El método de Langley está basado en el principio de extinción espectral y la ley de Beer- LambertBouguer. En este método las observaciones de radiación son ploteados como una función de la masa de aire donde la pendiente de la regresión lineal resultante es $\left(\mathbf{t}_{\text {total }}\right)$ y la radiación correspondiente a $m$ igual a cero es la $I_{\mathrm{o} \lambda}$.

El espesor óptico del aire, conocido como el de Rayleigh, es calculado utilizando la ecuación 3 (13):

$$
\tau_{R}(\lambda)=\left(a+b H \lambda^{-\left(c+d \lambda+\frac{e}{\lambda}\right)} \frac{P}{P_{S}}\right.
$$

Donde, $a=0,00864, \quad b=6,5^{*} 10^{-6}, \quad c=3,916$, $\mathrm{d}=0,074, \mathrm{e}=0,05 ; \mathrm{H}$ es la altura del fotómetro solar SP02-L en kilómetros; P es la presión de sitio ( 986 en mb); Ps, presión atmosférica estándar 1013,25 mb; y $\lambda$, la longitud de onda en micrómetros (13).

El espesor óptico de ozono está en función de la concentración de la columna total de ozono $(\Omega)$ en Unidades Dobson obtenido con el sensor OMI y el coeficiente de absorción de ozono $(\sigma)$ según la ecuación 4 .

$$
\tau_{O 3}=\frac{\Omega \times \sigma}{1000}(4)
$$

Para las longitudes de onda de 412, 500, 675 y 862, los coeficientes de absorción son $0.000295974375,0.031997375,0.0401001875$ y 0.00247309125 , respectivamente (14).

\section{RESULTADOS}

\section{Mediciones de intensidad incidente}

Para el procesamiento de datos en el cálculo del espesor óptico se realizaron lecturas cada 10 segundos, con cero cobertura nubosa alrededor del Sol, en un promedio de 82 lecturas por cada longitud de onda durante los meses de enero de 2007, enero de 2008 y enero-febrero de 2013.

Estudios realizados indican que al menos 20 ajustes son necesarios, debido a que variaciones monótonas en el EOA dan lugar a errores sistemáticos en la determinación de la intensidad inicial por el método de Langley (15), debido a que estos errores son imposibles de detectar en un solo ajuste (16). Por ello, para asegurar una adecuada se realizaron 7 ajustes a partir de los cuales se obtuvo un promedio, ajuste recomendado por otros investigadores (17), con la finalidad de determinar el EOA con un error absoluto de 0.01 - 0.02. En condiciones de baja turbidez este error absoluto puede significar un error relativo superior al $10 \%$ en el EOA (18).

En la obtención de la curva de Langley consiste en obtener medidas de voltajes con una variación continua de la columna de masa de aire entre el fotómetro solar y el sol. Y para la obtención de la intensidad en la parte superior de la atmósfera se hizo una extrapolación de la señal espectral para la obtención de la constante cuando la masa del aire $(m)$ es cero $(m=0)$, de acuerdo a la ecuación 5, para las longitudes de onda de 412, 500, 675 y $862 \mathrm{~nm}$ como se muestra en el gráfico $\mathrm{N}^{\circ} 1$,

$$
\ln \left(I(\lambda) R^{2}\right)=\ln \left(I_{0}(\lambda)\right)-m \tau(\lambda) \ldots \ldots(5)
$$



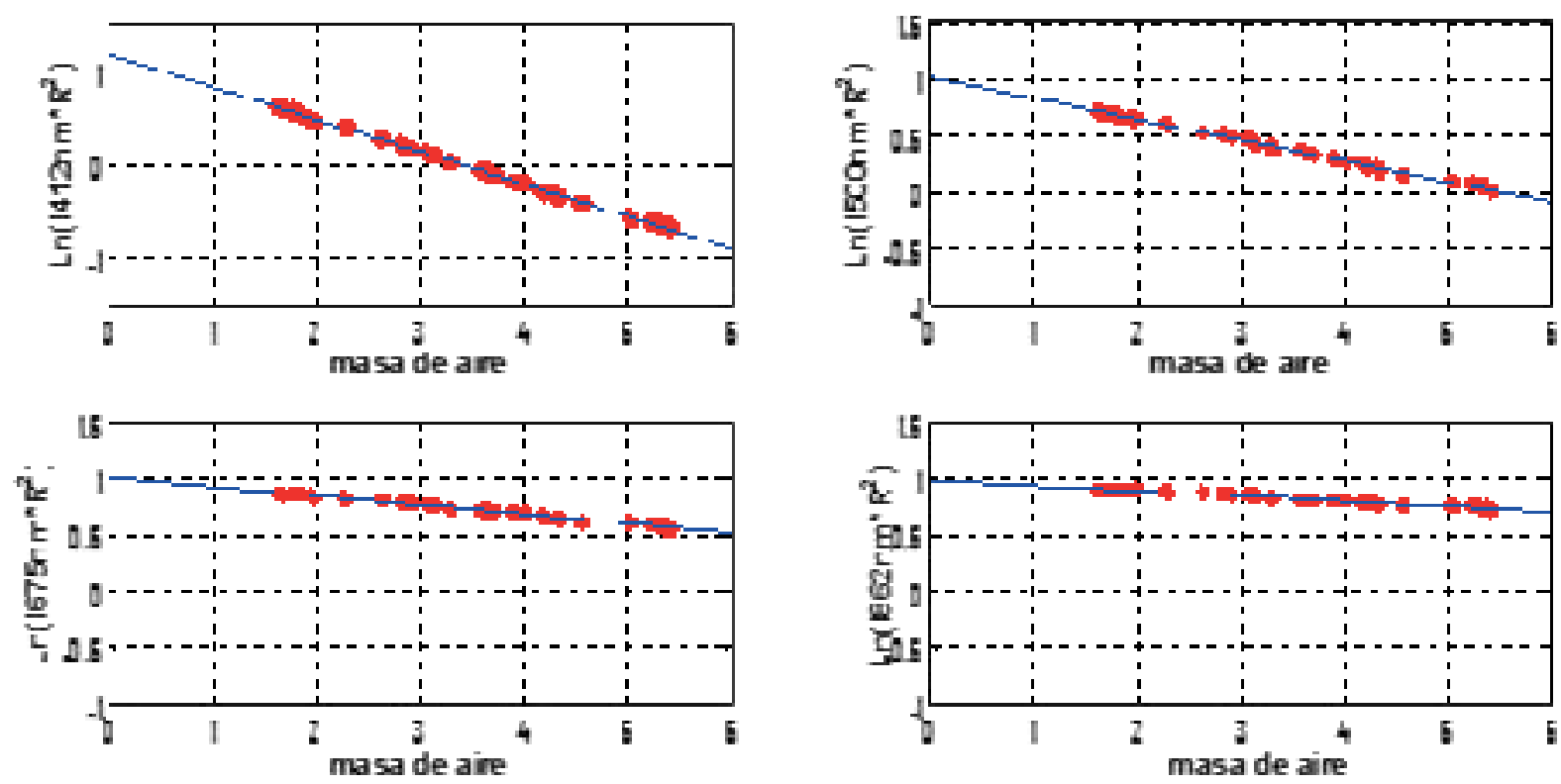

Gráfico 1: Grafico esquemático de la curva de Langley-plot para la obtención de la intensidad en la parte superior de la atmósfera

Así, se obtiene las intensidades en la parte superior de la atmósfera que representan la radiación incidente para una atmósfera limpia sin contaminantes obteniendo las intensidades iniciales de $3.2143,2.7661,2.7570$ y 2.6836 Voltios para las longitudes de onda 412, 500, 675 y $862 \mathrm{~nm}$, respectivamente, que nos indica la radiancia que incide cuando la masa del aire es cero.

\section{Espesores ópticos de ozono y Rayleigh}

En relación con los espesores ópticos, la Tabla $\mathrm{N}^{\circ} 1$ muestra un resumen de los valores encontrados para cada tipo de espesor óptico, del total de las observaciones realizadas con ángulos cenit solar inferior a $80^{\circ}$. Estos valores son normalmente pequeños en comparación con otras ubicaciones no polares, las condiciones de clima y ubicación de la región antártica condicionan a que estas propiedades ópticas sean menores (19).

Tabla 1: Espesor óptico de rayleigh y ozono

\begin{tabular}{lllll} 
Longitud de onda & $412 \mathrm{~nm}$ & $500 \mathrm{~nm}$ & $675 \mathrm{~nm}$ & $862 \mathrm{~nm}$ \\
\hline Espesor óptico de ozono & 0,000078911 & 0,0085 & 0,0107 & 0,0006593 \\
\hline Espesor óptico de Rayleigh & 0,2367 & 0,1154 & 0,0373 & 0,0148 \\
\hline
\end{tabular}


Espesor óptico de aerosol determinado con el SP02-L

El gráfico $\mathrm{N}^{\circ} 2$ corresponde a las mediciones promedio de los años 2007, 2008 y 2013, con cero de cobertura solar durante las horas de la mañana, en la cual muestra un resumen de los valores encontrados para cada longitud de onda del espesor óptico del total de las observaciones realizadas con una masa de aire óptica de 2 a 5 , se puede observar que el espesor óptico de aerosol disminuye a medida que aumenta la longitud de onda mientras que la interacción entre la radiación y los aerosoles se incrementa, siendo la longitud de onda de $862 \mathrm{~nm}$ la que tiene mayor variación por efecto de los aerosoles donde el valor promedio y coeficiente de variación de EOA a $500 \mathrm{~nm}$ y 862 son de $0,07940,0144$ y 0,0493 0,0187 respectivamente. Estos resultados de espesor óptico de aerosol (EOA) están dentro del rango de los hallados en regiones polares por otros grupos (19), siendo menores en relación con los encontrados en ciudades urbanas entre 0,25 a 1,7 (20) y mucho menores que los registrados en lugares de quema de la biomasa donde llegan a valores en el rango de 0,6 a 2,4 para similares longitudes de onda (21).

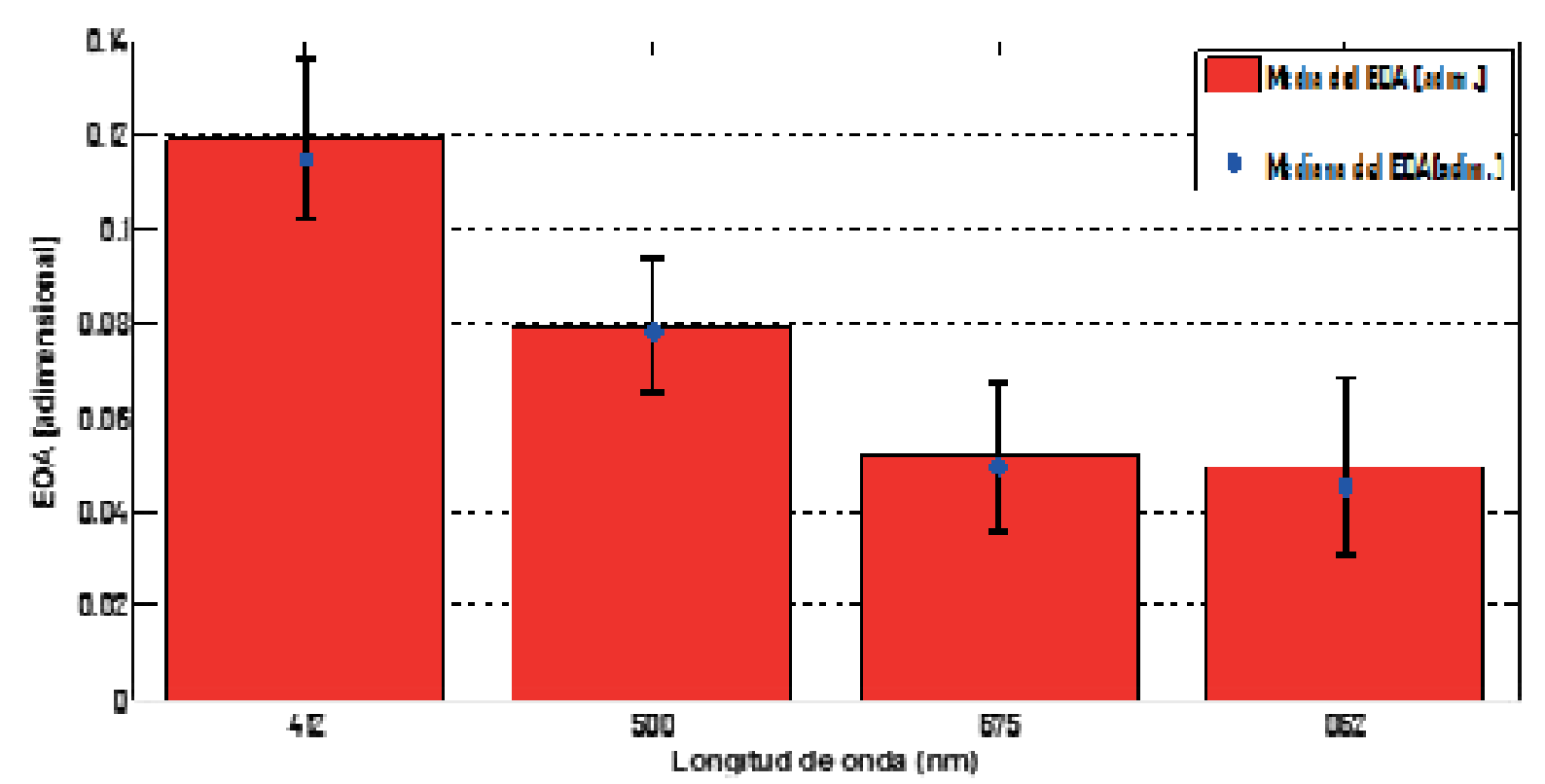

Gráfico 2: Variación del espesor óptico para cada longitud de onda

En el gráfico $\mathrm{N}^{\circ} 3$ se muestra una serie histórica de los valores del EOA durante 2007, 2008 y 2013. Como se puede observar la mediana del EOA varia entre 0,0641 a 0,1052 , siendo típico por las condiciones de turbidez atmosférica del sitio en este tiempo del año (22). La campaña fue planificada con el objetivo principal de cuantificar la comparación que existe entre los distintos años de mediciones donde se dio una máxima y mínima media de EOA a $500 \mathrm{~nm}$ de 0,1061 y 0,0646 para el 14 y 23 de enero del 2007, respectivamente. 


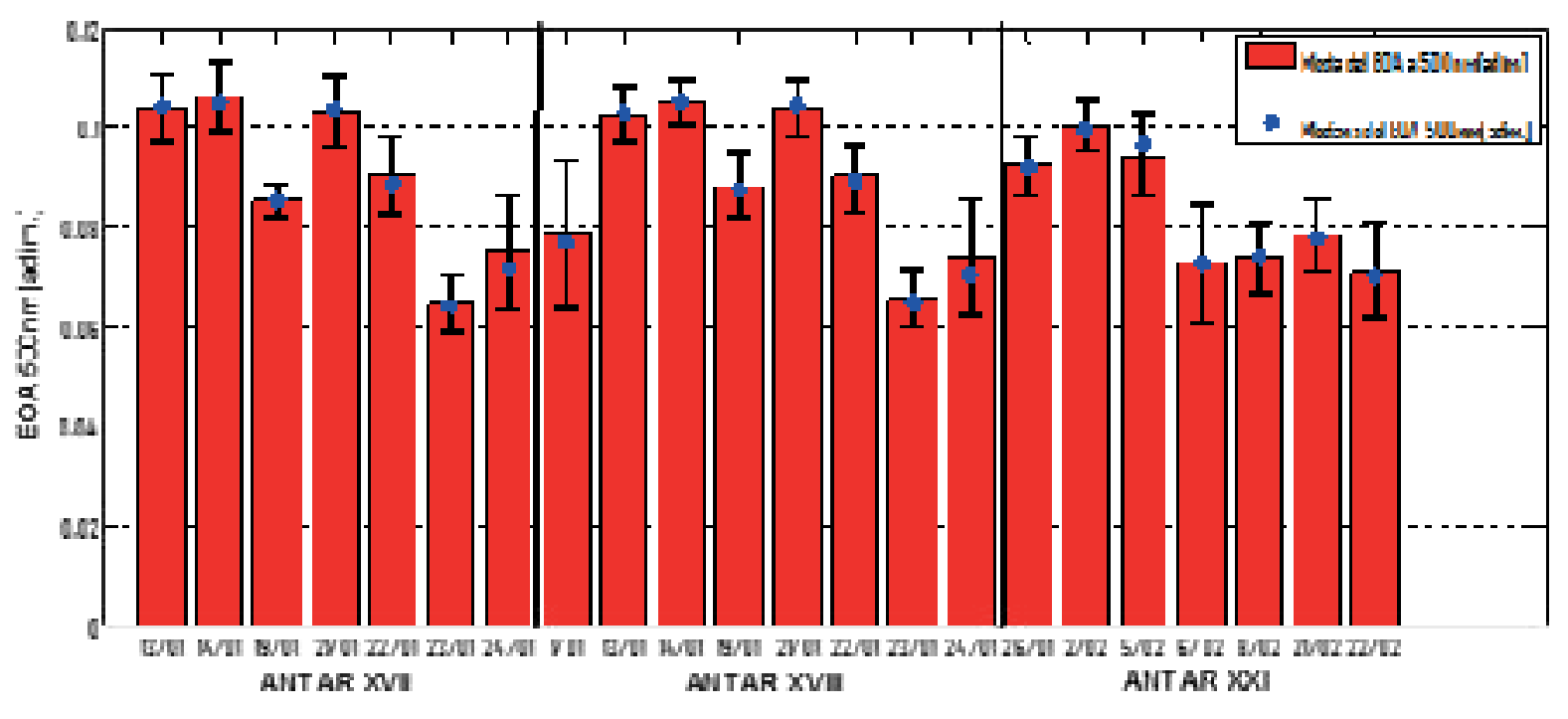

Gráfico 3: Variación diaria del EOA a 500 nm para los años de 2007, 2008 y 2013

Comparación de espesores ópticos de aerosol obtenidos con el sensor MODIS y SP02

El gráfico $\mathrm{N}^{\circ} 4$ corresponde a las mediciones de los años 2007, 2008 y 2013, para el sensor MODIS a $550 \mathrm{~nm}$ que pertenece a los satélites Aqua y Terra, y para el fotómetro solar SP02-L a 500 $\mathrm{nm}$, cuyo proceso se comparó ambas mediciones para los 3 años y quedó determinado que el grado de correlación entre el sensor MODIS (per- teneciente a los satélites Aqua y Terra) y el fotómetro solar SP02 es bajo, de 0,0284 y 0,0059, respectivamente, por el que se recomienda una estudio detallado del sensor MODIS. En cambio, en la comparación del espesor óptico de aerosol (EOA) que se determinó con el fotómetro solar SP02 a 500 nm, y el Índice de Aerosol (IA) que se obtuvo mediante el sensor OMI a $360 \mathrm{~nm}$ se determinó un coeficiente de correlación de 0,3606 con un $p$-value $<0,05$.

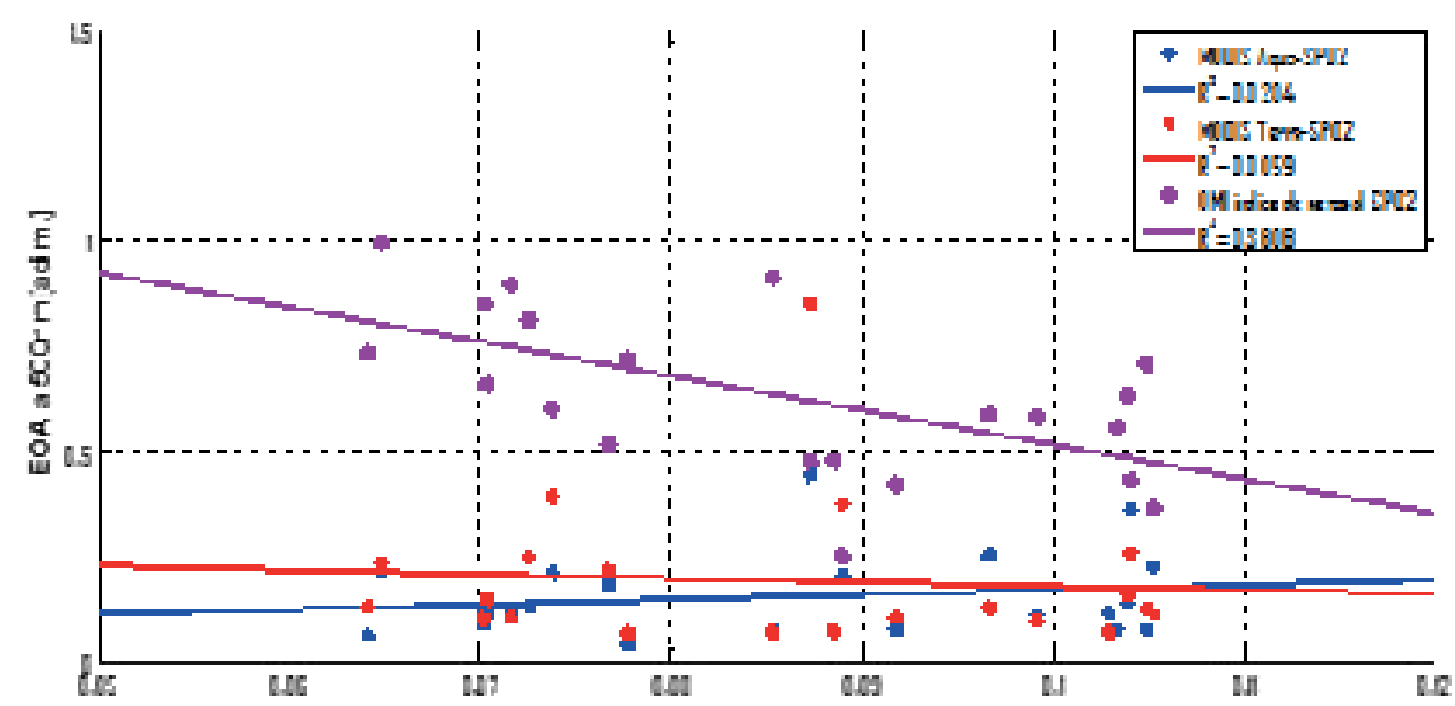

Gráfico 4: Comparación entre datos obtenidos con los sensores MODIS y OMI con el fotómetro solar SP02-L. 


\section{DISCUSIÓN}

En el gráfico $\mathrm{N}^{\circ} 5$ considerando la estación antártica peruana, la ECAMP presenta una alta mediana de EOA de 0,0781 como las estaciones de Neumayer y Aboa de 0,06 y 0,0551, respectivamente a $500 \mathrm{~nm}$, ya que pertenecen a la zona costera donde se da mayor influencia de aerosoles marinos. En cambio, los que no pertenecen a la zona costera presentan valores mínimos como la estación de Kohnen y South Pole de 0,015 cada una (22).

La comparación entre la ECAMP con las otras estaciones ofrece la evidencia de las diferencias considerables de las propiedades ópticas de los aerosoles. Es cierto que el aire es limpio en regiones polares, pero tienen una mezcla de partículas muy pequeñas principalmente de origen marino y a las condiciones de turbidez que suelen aplicar en verano y en otoño los fuertes cambios debidos al transporte de bruma y polvo (23).

El impacto radiativo directo de los aerosoles polares en la superficie y en la parte superior de la atmósfera necesitan ser estudiados más detenidamente a través de estudios teóricos sobre las propiedades radiativas de los aerosoles. La verificación de los resultados teóricos requiere el monitoreo continuo de las propiedades ópticas de los aerosoles, como determinar los modelos más realistas que sean de reflectancia anisotropía de la superficie para representar las diferentes albedos de superficie y características del mar y tierra en las regiones polares, como lo definió Ricchiazzi (24), también es necesario el factor de calibración para la determinación del EOA como las correcciones del espesor óptico de Rayleigh (25).

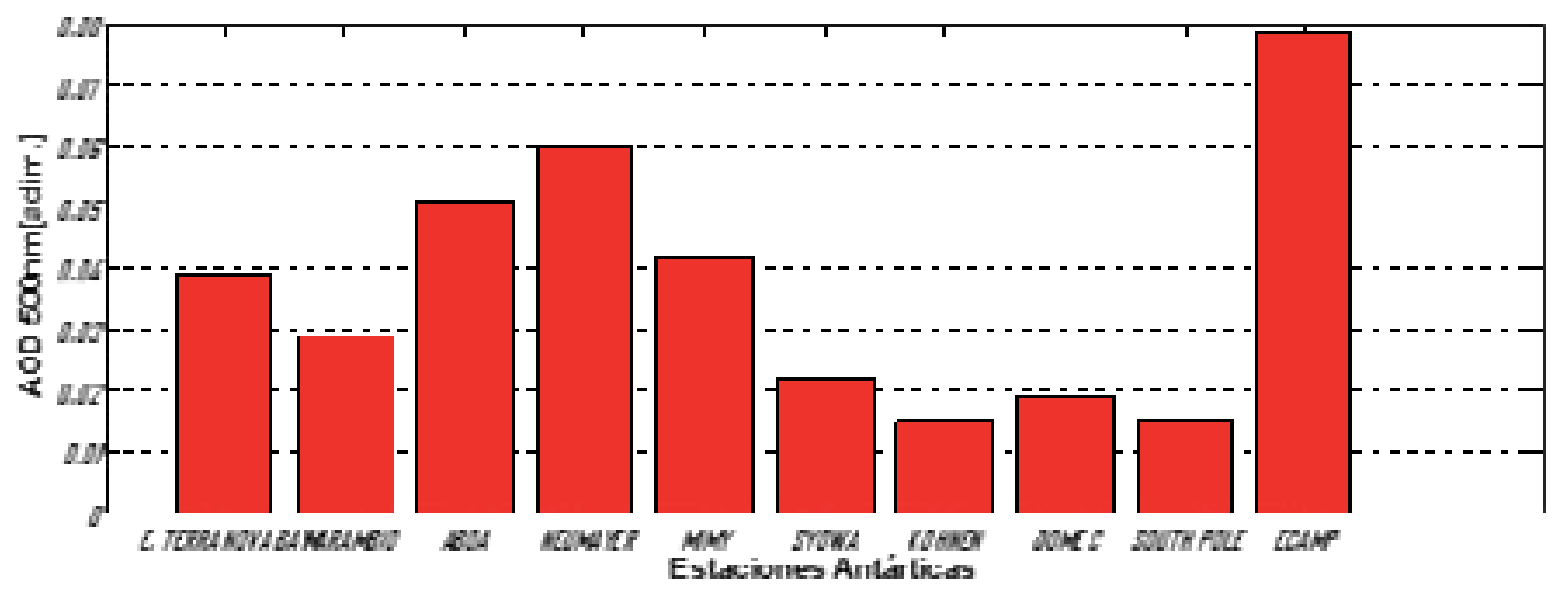

Gráfico 5: Variación del AOD a 500 nm de las estaciones antárticas

El trabajo permitió cuantificar el EOA en la ECAMP, con la finalidad de proveer y contribuir al estudio del forzamiento radiativo y a las propiedades ópticas de los aerosoles en zonas polares, e implementar estaciones de monitoreo continuo del EOA para realizar comparaciones con los datos satelitales, y obtener una nueva metodología para la obtención del EOA por sensoramiento remoto por satélite, ya que las in- certezas en los espesores ópticos obtenidos por sensores en satélites depende fuertemente de la calidad de la información sobre las propiedades ópticas de las partículas de los aerosoles, ya que en la presente investigación dio valores muy elevados de espesores ópticos obtenidos con el sensor MODIS, en relación a los valores medidos con el fotómetro solar en superficie. 


\section{AGRADECIMIENTO}

Agradecimientos al Instituto Antártico Peruano por su apoyo en la realización de las mediciones y elaboración del presente artículo como parte de la expedición antártica.

\section{REFERENCIAS BIBLIOGRÁFICAS}

1. Vergaz B. Propiedades ópticas de los aerosoles atmosféricos. Caracterización del área del Golfo de Cádiz, Valladolid. [Tesis Doctoral]: Universidad de Valladolid; 2001.

2. Foster P, Ramaswamy. IPCC, Changes in atmospheric constituents and in radiative forcing. UNIVERSITY PRESS CAMBRIDGE. NEW YORK, USA; 2007.

3. Andreae M. Climatic effects of changing atmospheric aerosol levelsen World Survey of Climatology. Elsevier.1995; 16: 341-392.

4. Raes, Frank, Dingenen R, Vignati E, Wilson J, Putaud J, Sinfeld J y Adams M. Formation and cycling of aerosols in the global troposphere. Atmos. Environ. 2000; 34: 4215-4240.

5. WMO. Report of the WMO.WMO aerosol measurement procedure; 2003

6. Ostro B, Eskeland G, Sánchez J. y Feyzioglu T. Air Pollution and Health Effects: A Study of Medical Visits among Children in Santiago, Chile. Environmental Health Perspectives. 1999; 107 (1):69-73.

7. Ostro B, Sánchez J, Aranda C y Eskeland G. Air Pollution and Mortality: Results from a Study of Santiago, Chile. Journal of Exposure Analysis and Environmental Epidemiology. 1996; 6(1): 97-114.

8. Kirchhoff V, Silva A, Costa C, PesLeme N, Pavao $\mathrm{H}$, Zarratti F. UV-B optical thickness observations of the atmosphere. Journal of Geophysical Research. 2001; 106: 2963-2973.

9. Tomasi C, Vitale V. Calculation of the relative optical mass functions for air, water vapor, ozone and nitrogen dioxide in the Antarctic and Arctic Atmospheres. 7th Workshop Italian Research on Antarctic Atmosphere, Conference Proceedings. Italian Physical Society, Bologna, Italy. 1997; 62: 22-24.
10. Guía de usuario del fotómetro solar modelo SP02L de Middleton Imc; 2004

11. Bodhaine B, Wood N, Dutton E, and Slusser J. On rayleigh Optical Depth Calculations. Journal Atmos. and Ocean. 1999; 16: 1854-1864.

12. Reagan J, Scott - Fleming I., Herman BySchotland R.Recovery of spectral optical, depth and zero - air mass solar spectral irradiance under conditions of temporally varying optical depth proceedings of IGARSS'84 Symposium. Strasbourg. 1984; 215: 455-459.

13. Liou K. An introduction to atmospheric radiation. $2^{\text {da }}$ ed. New York: academic Press; 2007.

14. Michalsky J. Aerosol optical depth value-added product. Climate Research. 2013; 129: 1-32.

15. Reagan J, Thomason L, Herman B, Palmer J. Assessment of atmospheric limitations on the determination of the solar spectral constant from ground-based spectroradiometer measurements. Geosci. Remote. 1986; 24: 258-265.

16. Terez E, Terez G. A method to determine atmospheric optical depth using observations of direct solar radiation. J. Geophys. 2003; 108 (D22):1-6.

17. Slusser J, Gibson J, Bigelow D. ,Kolinski D, Disterhoft P, Lantz K, Baubien A. Langley method of calibrating UV filter radiometers. J. Geophys. 2000; 105 (D4): 4841-4849.

18. Wehrli Ch. Calibration of filter radiometers for determination of atmospheric optical depth. Metrologia. 2000; 37 (5): 419-422.

19. Stone R. Monitoring aerosol optical depth at Barrow, Alaska and South Pole; Historical overview, recent results, and future goals. Coop. Res. in Environ. Scien. 2002; 80: 123-144.

20. Castro T, Madronich S, Rivale S, Muhlia A, Mar B. influence of aerosols on photochemical smog in Mexico City. Atmospheric Environment. 2001; 35: 1765-1772.

21. Eck T, Holben B, Reid J, O’Neill N, Schafer J, Dubovik O, Simimov A, Yamasoe M y Artaxo P. High aerosol optical depth biomass burning events: A comparison of optical properties for different source regions. Geophysical Research Letters. 2003; 30(20): 2035-2044. 
22. Dutton E, Reddy P, Ryan S, Tomasi C. Aerosol in polar regions; A historical overview based on optical depth and in situ observations, J. Geophys. Res. 2007; 112: 1-28.

23. Shaw, G. Atmospheric turbidity in the Polar Regions. J. Appl. Meteorol. 1982; 21: 1080- 1088.

24. Ricchiazzi P, Gautier C. The effect of non-Lambertian surface reflectance on aerosol radiative forcing. U.S. Dep. of Energy Daytona Beach. 2005; 14- 18.

25. Mazzola M, Stone R. Evaluation of sun photometer capabilities for retrievals of aerosol optical depth at high latitudes: The POLAR-AOD intercomparison campaigns. Atmos. Environ. 2012; 52:4-17. 